\title{
Caída de tefra y su influencia sobre la estructura y dinámica de los bosques andinos de Nothofagus en el Parque Nacional Puyehue, Chile
}

\author{
Tephra fall and its influence on the structure and dynamics of \\ Andean Nothofagus forests in Puyehue National Park, Chile
}

\author{
Mauricio Montiel ${ }^{1,2,3}$, Mauro E. González ${ }^{2,3}$ \& Charles M. Crisafulli ${ }^{4}$
}

Los grandes disturbios tienen un rol fundamental en la dinámica de los bosques de Nothofagus a través de la generación de grandes aperturas del dosel que crean condiciones ambientales espacialmente heterogéneas para el posterior establecimiento de la regeneración (González et al. 2014). En el caso del vulcanismo, uno de estos agentes de grandes disturbios, si bien se reconoce su importancia en la dinámica de los bosques andinos (Veblen, 1985), el papel de la ceniza volcánica como impulsor de establecimiento $y$ agente de mortalidad en estos bosques ha sido escasamente estudiado (e.g. Vogel, 1996).

La reciente erupción del Complejo Volcánico Puyehue- Cordón Caulle (CVPCC) en junio del 2011, tuvo por resultado el depósito de grandes cantidades de ceniza volcánica (tefra), afectando fuertemente la vegetación en una amplia zona geográfica. Durante el siglo XX el CVPCC ha tenido varias erupciones, destacando aquellas de 1921 - 1922 (diciembre a febrero de 1922) y la de 1960 , cuyo material fue depositado principalmente en sentido oeste-este, impactando consecutivamente los bosques ubicados al sureste del complejo volcánico (González-Ferrán, 1995). En este contexto, la presente nota científica apunta a comprender la influencia de las sucesivas caídas de tefra en el origen y desarrollo de los bosques dominados por Nothofagus en el entorno del CVPCC.

Muestreo de terreno y análisis de datos

La investigación se realizó en bosques andinos dominados por Nothofagus dombeyi (Mirb.) Bl. y Nothofagus pumilio (Poepp. et Endl.) Krasser, ubicados en el Parque Nacional Puyehue, específicamente en el sector fronterizo del paso Cardenal Samoré o paso Puyehue $\left(40^{\circ} 41^{\prime} 52^{\prime \prime} \mathrm{S}\right.$, 71'57'34” O). Tras la erupción del CVPCC en junio del 2011, estos bosques fueron afectados por la caída de un manto de tefra de aproximadamente $50 \mathrm{~cm}$ de espesor (Novoa-Melson 2013; Fig. 1). El área de estudio posee un clima templado lluvioso de montaña con una precipitación promedio anual superior a $5000 \mathrm{~mm}$, predominando los vientos del norte, noroeste y oeste durante la mayor parte del año debido a la influencia de los frentes polares (Godoy et al. 1999).

En el área de estudio se seleccionaron tres rodales que incluyeron un bosque mixto de $N$. dombeyi- N. pumilio (BM) ubicado a $1090 \mathrm{~m}$ s.n.m., y dos bosques puros de $N$. pumilio (BP1 y BP2) ubicados entre 1300 y 1315 m.s.n.m.

1 Universidad Austral de Chile, Facultad de Ciencias Forestales y Recursos Naturales, Escuela de Graduados, Valdivia, Chile; mauricio.montiel711@gmail.com

2 Universidad Austral de Chile, Facultad de Ciencias Forestales y Recursos Naturales, Instituto de Conservación Biodiversidad y Territorio, Laboratorio de Ecología de Bosques, Valdivia, Chile. $\bowtie$ maurogonzalez@uach.cl

3 Centro de Ciencia del Clima y la Resiliencia (CR) ${ }^{2}$, Valdivia, Chile.

4 United States Forest Service, Pacific Northwest Research Station, Washington, USA; ccrisafulli@fs.fed.us 


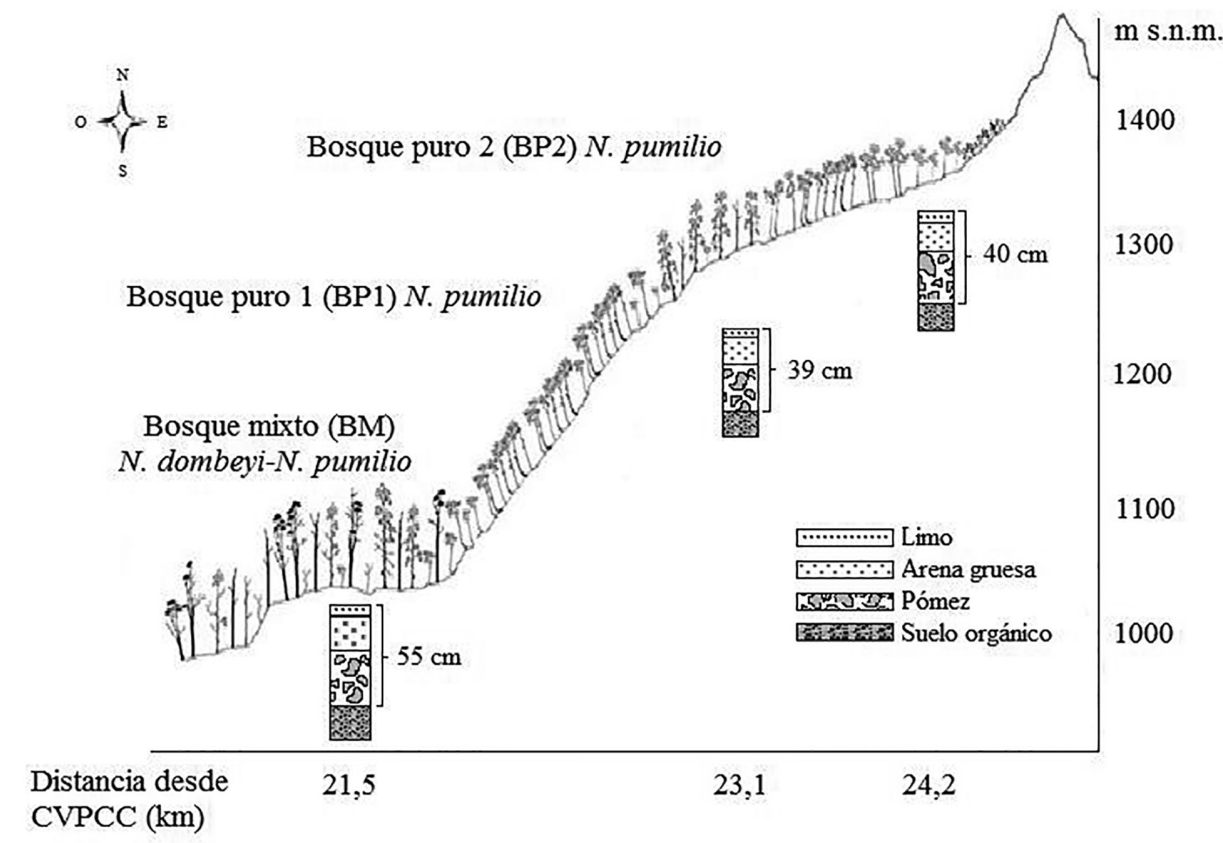

Fig. 1. Bosques subandinos de Nothofagus en el Parque Nacional Puyehue. Los perfiles de suelo muestran la variación en profundidad y la composición de los depósitos de tefra (Novoa-Melson 2014) asociados a la variación topográfica.

En cada uno de los rodales se establecieron dos parcelas circulares de $1000 \mathrm{~m}^{2}$ para registrar todos los árboles remanentes ( $\geq 45 \mathrm{~cm}$ de DAP) y una parcela circular anidada dentro de ella de $500 \mathrm{~m}^{2}$ en donde se registraron todos los árboles $\geq 5 \mathrm{~cm}$ de DAP. La posición sociológica de los árboles se determinó siguiendo la clasificación de Kraft (Donoso, 1993). Para determinar el período de establecimiento de los bosques mixtos (BM), más heterogéneos estructuralmente, a cada individuo en la parcela se le extrajo un tarugo de incremento. En el caso de los bosques puros de N. pumilio (BP1 y BP2), debido a la alta densidad de individuos sólo fue necesario la extracción de tarugos desde la mitad de la parcela anidada para obtener un mínimo de 30 muestras $\left(250 \mathrm{~m}^{2}\right)$. Los tarugos fueron extraídos a una altura promedio de $16 \mathrm{~cm}$ sobre el nivel de la tefra. Con la finalidad de tener una referencia de los anillos faltantes entre la altura a nivel de tefra $(\mathrm{ca} .50 \mathrm{~cm})$ y la base del árbol (a nivel suelo orgánico), se excavó en la ceniza para extraer tarugos a ca. $10 \mathrm{~cm}$ de altura a seis individuos. Para verificar la ocurrencia de incendios en el área de estudio, se colectaron cicatrices en cada uno de los rodales según las técnicas y protocolo de Arno y Sneck (1977). Los tarugos y cicatrices colectadas fueron preparados de acuerdo al método propuesto por Stokes \& Smiley (1968) para luego fechar los anillos de crecimiento bajo lupa estereoscópica. Para las muestras analizadas que no contenían la médula, el número de anillos faltantes se estimó con el método geométrico de Duncan (1989). Los tarugos en que no fue posible realizar dicha estimación fueron considerados como edad mínima, siempre que el radio del tarugo fuera $>75 \%$ respecto del radio calculado del árbol.

\section{Establecimiento, estructura y composición arbórea}

La erupción volcánica del CVPCC del 2011 tuvo un significativo efecto en la mortalidad de los bosquesandinos de Nothofagus spp., especialmente aquellos ubicados en terrenos de menor pendiente $y$ con una mayor profundidad de tefra $(>50 \mathrm{~cm})$. En el BM, el área basal total de árboles muertos de N. dombeyi se estimó entre 11,8 y $17,4 \mathrm{~m} 2 \mathrm{ha}^{-1} \mathrm{y}$ en $N$. pumilio entre 12,8 y $29,8 \mathrm{~m}^{2}$ ha $^{-1}$ (Tabla 1 ). Esto significó un área basal de árboles muertos de más de un 55 \% en el rodal que, indistintamente de 
Tabla 1. Área basal de Nothofagus por rodal.

\begin{tabular}{|c|c|c|c|c|c|c|c|c|}
\hline \multicolumn{9}{|c|}{ Área basal $\left(\mathrm{m}^{2} \mathrm{ha}^{-1}\right)$} \\
\hline \multirow[b]{2}{*}{ Rodal } & \multirow[b]{2}{*}{ Parcela } & \multicolumn{2}{|c|}{ N. dombeyi } & \multicolumn{2}{|c|}{ N. pumilio } & \multirow{2}{*}{$\begin{array}{c}\text { Nothofagus spp. }{ }^{* *} \\
\text { muerto }\end{array}$} & \multicolumn{2}{|c|}{ Total } \\
\hline & & vivo & muerto & vivo & muerto & & vivo & muerto \\
\hline \multirow{2}{*}{$\mathrm{BM}$} & A & $15(25,1)^{*}$ & $17,4(29,1)$ & $11,8(19,7)$ & $12,8(21,4)$ & $2,8(4,7)$ & $26,8(45)$ & $33(55)$ \\
\hline & B & $16,8(25,3)$ & $11,8(17,8)$ & $7,6(11,4)$ & $29,8(44,9)$ & $0,4(0,6)$ & $24,4(37)$ & $42(63)$ \\
\hline \multirow{2}{*}{$\mathrm{BP} 1$} & $\mathrm{C}$ & & & $38,3(85,5)$ & $6,5(14,5)$ & & $38,3(85)$ & $6,5(15)$ \\
\hline & $\mathrm{D}$ & & & $58(94.7)$ & $3,2(5.3)$ & & $58,0(95)$ & $3,2(5)$ \\
\hline \multirow{2}{*}{ BP2 } & E & & & $71,9(97,3)$ & $2(2,7)$ & & $71,9(97)$ & $2(3)$ \\
\hline & $\mathrm{F}$ & & & 80,9 (95.9) & $3,5(4,1)$ & & $80,9(96)$ & $3,5(4)$ \\
\hline
\end{tabular}

* Entre paréntesis porcentaje que representa en relación al total.

** Nothofagus no identificados debido al deterioro de la corteza.

su edad y diámetro, murieron a consecuencia del daño mecánico provocado por la caída de tefra. Al observar los anillos de crecimiento de los árboles aún sobrevivientes que poseen una muy baja masa foliar, muestran que algunos individuos no formaron anillos luego de la erupción (Montiel, 2014), lo que sugiere que la mortalidad de Nothofagus spp. seguirá incrementándose en este sitio.

Por otra parte, la curva de distribución de tamaños en BM describe una curva exponencial negativa que se caracterizó por la presencia de individuos en todas las clases de tamaño (Fig. 2). Además el BM muestra individuos remanentes de una cohorte establecida durante el período post1850, con un claro pulso de establecimiento de una cohorte más joven a partir de la década de 1920. Estos rodales incluyen árboles remanentes de gran diámetro establecidos desde los 1600 (Fig. 2)

La estructura de tamaños y de edades de los bosques secundarios corresponde a la típica curva normal que caracteriza a rodales coetáneos establecidos luego de un disturbio de gran intensidad (Figs. 3 y 4). En este caso los árboles muertos en pie corresponden a individuos rezagados en su crecimiento debido a la intensa competencia, formando parte de la misma cohorte de aquellos árboles actualmente vivos, lo que puede verificarse por el avanzado estado de deterioro que presentan, con ausencia total de corteza, a diferencia de aquellos individuos muertos debido a la caída de tefra en el BM. En ambos rodales (BP1 y BP2) el área basal de los árboles vivos representó más del $80 \%$ del total (Tabla 1). Esta baja mortalidad se debió principalmente a una menor cantidad de tefra depositada ( $40 \mathrm{~cm}$; Fig. 1$)$ y a la posterior recuperación y aumento de la cobertura foliar en N. pumilio (Novoa-Melson, 2013)

De manera similar al bosque mixto, los bosques secundarios presentaron una densa cohorte post-disturbio de $N$. pumilio en la década de 1920. Se observa también la presencia de árboles remanentes de N. pumilio establecidos entre 1680 y 1840 , e individuos establecidos desde los 1850 que corresponderían a individuos sobrevivientes de una cohorte que fue afectada por el disturbio que a su vez promovió el establecimiento del 1920 (Fig. 3). Este masivo establecimiento de N. pumilio se presume ocurrió posterior a la depositación de tefra luego de la erupción del CVPCC en 19211922 (indice de explosividad volcánica $=3$; Lara et al. 2006) que probablemente produjo una gran mortalidad de individuos en el bosque. En el caso de BP1, la presencia de numerosos troncos caídos y desraizados ( $>45 \mathrm{~cm}$ de DAP) en dirección a la pendiente sugieren además una caída masiva por viento de la cohorte previa, luego de la cual se estableció una cohorte de N. pumilio bajo un dosel relativamente abierto de árboles de Nothofagus spp. remanentes. Este disturbio por viento parece 

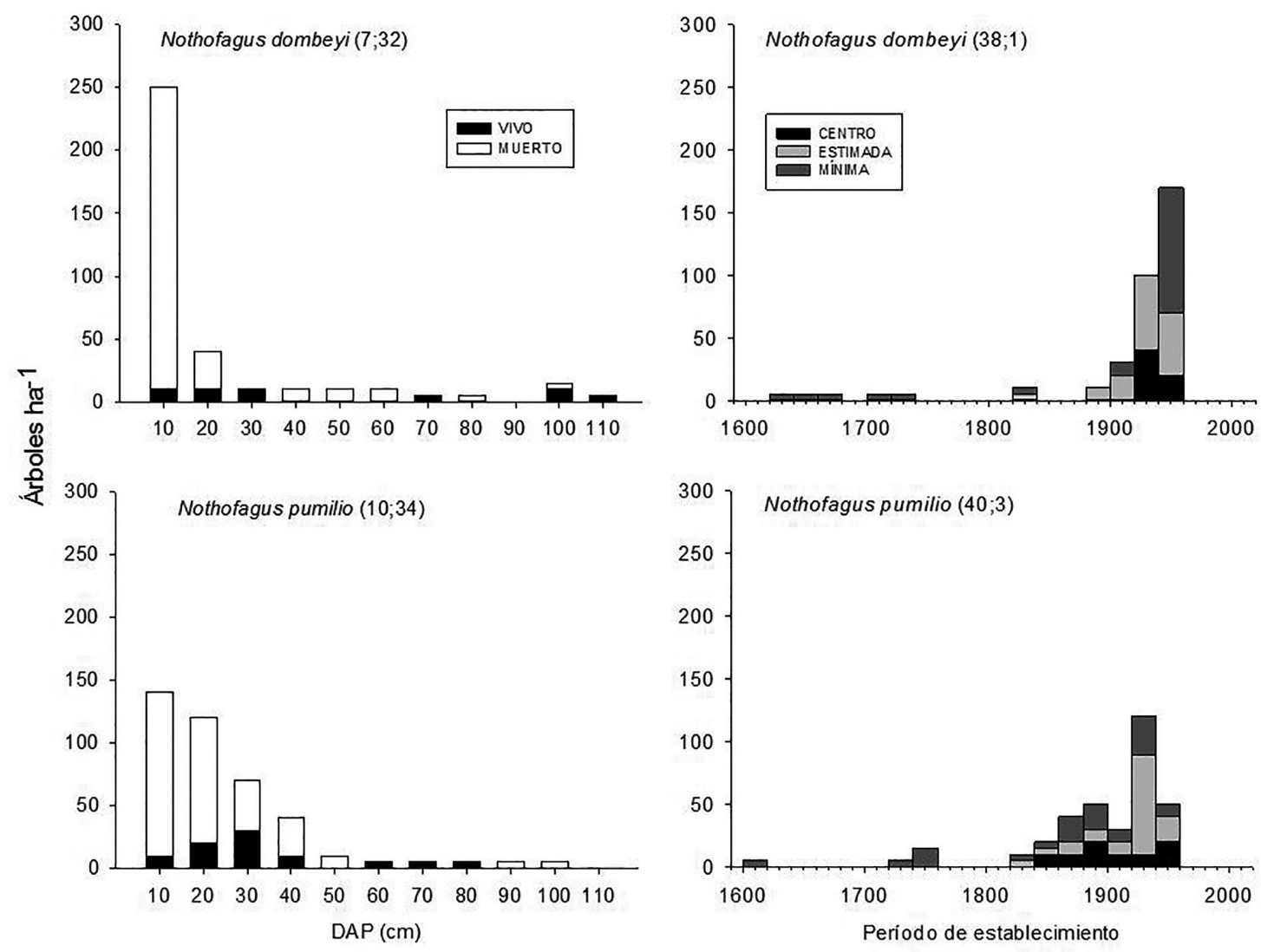

Fig. 2. Estructura de tamaño y período de establecimiento del bosque mixto (BM). Para la distribución de tamaños, los valores entre paréntesis indican el número de árboles vivos y muertos. Para la distribución de edades, los valores entre paréntesis indican el número de árboles tarugados y el número de árboles excluidos.
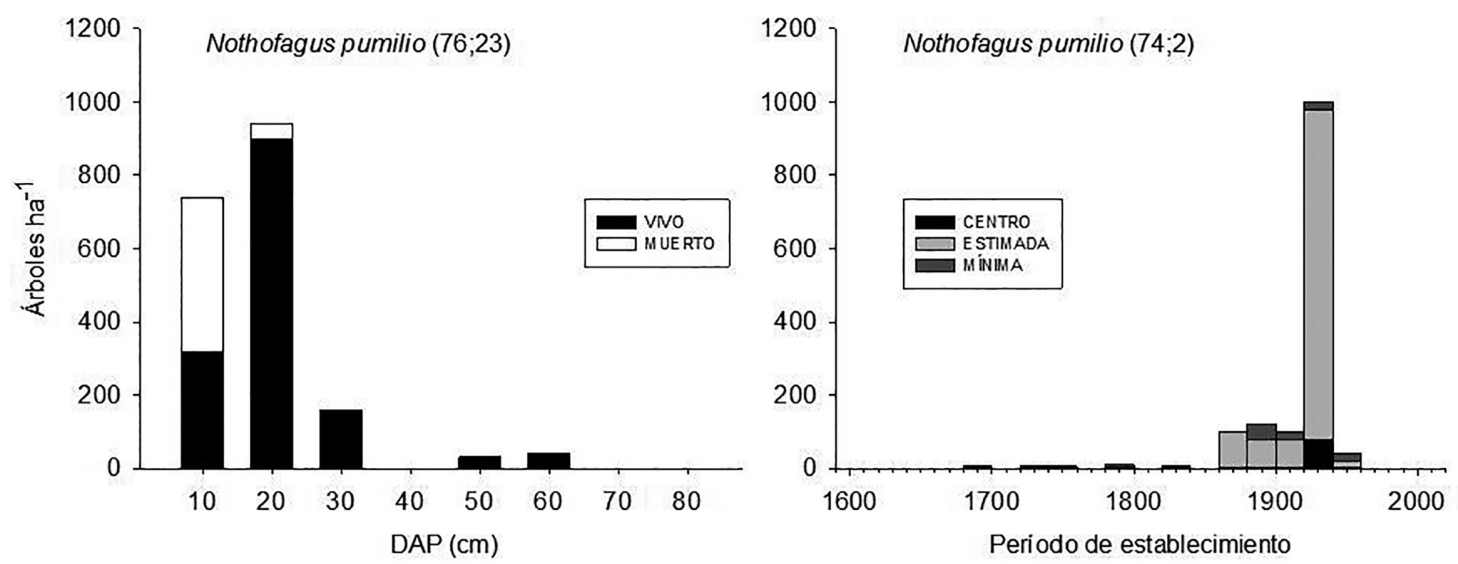

Fig. 3. Estructura de tamaño y período de establecimiento del bosque en BP1. Para la distribución de tamaños, los valores entre paréntesis indican el número de árboles vivos y muertos. Para la distribución de edades, los valores entre paréntesis indican el número de árboles tarugados y el número de árboles excluidos. 

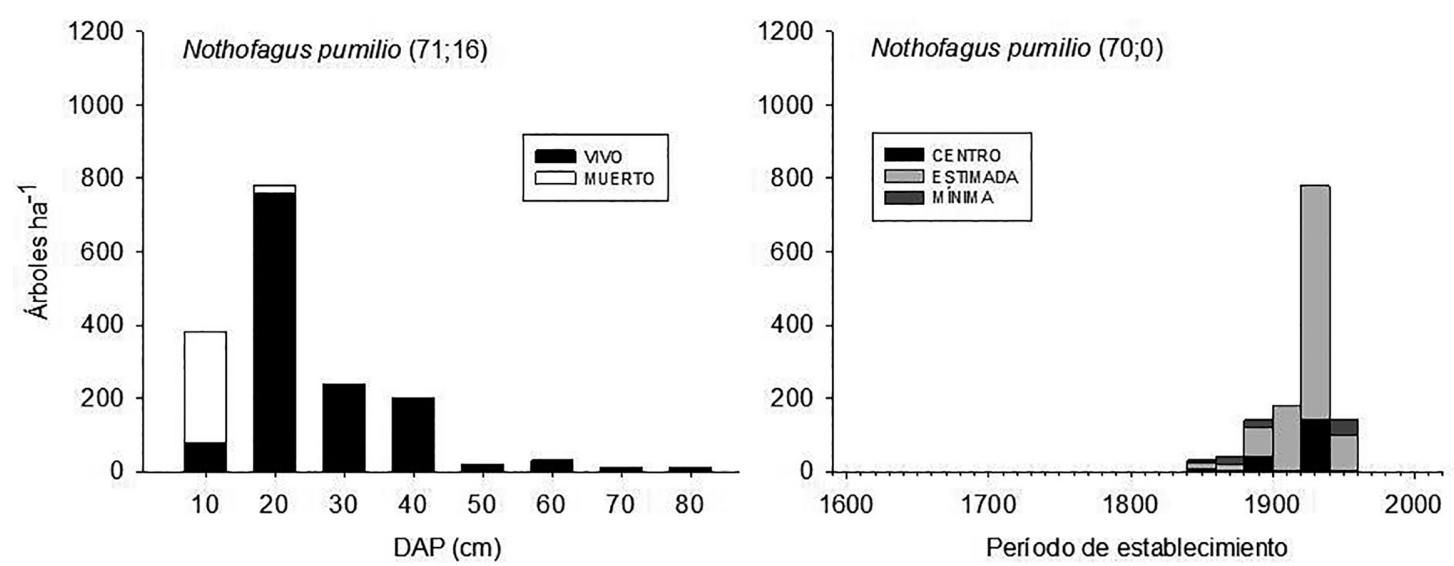

Fig. 4. Estructura de tamaño y período de establecimiento del bosque en BP2. Para la distribución de tamaños, los valores entre paréntesis indican el número de árboles vivos y muertos. Para la distribución de edades, los valores entre paréntesis indican el número de árboles tarugados y el número de árboles excluidos.

haber antecedido a la erupción del CVPCC en 1921-22 y por tanto la nueva cohorte establecida aprovechó tanto la apertura originada por la caída por viento como el nuevo sustrato de ceniza volcánica y legados biológicos disponibles (Franklin et al. 2000). Estas observaciones sugieren que los cambios demográficos asociados a la mortalidad de individuos producto de perturbaciones previas y la posterior caída de tefra promueven el rápido establecimiento y el éxito de la regeneración arbórea, aun cuando este sustrato inicialmente sea pobre en nutrientes (Novoa-Melson, 2013).

El rápido y denso establecimiento de plántulas post erupción de Nothofagus spp.es característico de sitios perturbados por la caída de tefra. Observaciones realizadas por Vogel (1996) tras la erupción del Volcán Hudson en 1991 dan cuenta del masivo establecimiento de Nothofagus a partir del segundo año luego de la erupción. Tras la última erupción del CVPCC en 2011, también fue posible observar una muy abundante regeneración de Nothofagus spp. incluso bajo la cobertura de los rodales coetáneos; sin embargo, aún no ha sido posible establecer la tasa de sobrevivencia y el probable carácter efímero de dicha regeneración.

Por otra parte, luego de la erupción del CVPCC en 1960, la cantidad de material eyectado no fue lo suficiente como para provocar un cambio en el ecosistema similar al de los años 1920s y, por lo tanto, en el establecimiento de una nueva cohorte en los sitios estudiados. Además, las condiciones climáticas en la cual se desarrolló la erupción eran de intensa lluvia, suficiente como para que la escorrentía removiera la ceniza (Wrigth \& Mella, 1963).

Las fechas determinadas en las cicatrices obtenidas en los rodales BP1 y BP2 resultaron asincrónicas, lo que sumado a la peculiar ubicación de la cicatriz en el fuste (fuste dañado desde la copa a la base en forma helicoidal) indicaría que este daño corresponde a la caída de rayos. En efecto, en esta zona ubicada en la parte norte de la Patagonia, las tormentas convectivas, que sólo son frecuentes durante los años en donde las masas de aire subtropicales húmedos penetran anómalamente hacia el sur en la región, están asociados con años de veranos cálidos en los cuales es frecuente la caída de rayos (Kitzberger \& Veblen, 2003). En el caso del BM, si bien en el fechado de cicatrices, se observó cierta sincronía entre las muestras esto no permitió establecer con claridad la ocurrencia de un incendio en el rodal.

En sintesis, la estructura y dinámica de los bosques de Nothofagus spp. andinos está importantemente relacionada a disturbios exógenos de gran escala como son las erupciones volcánicas y la caída masiva por viento. La sincronía en el pulso de establecimiento post-1920 sugeriría que tanto los bosques secundarios como mixtos fueron afectados por la caída de tefra de la erupción de 1921 - 1922 promoviendo un pulso de establecimiento de las especies de Nothofagus 
spp. Además, en el caso de los bosques puros de N. pumilio, ubicados a mayor altitud, la abundante presencia de cicatrices en el fuste de los árboles indican el frecuente impacto por la caída de rayos.

\section{AGRADECIMIENTOS}

Reciban nuestros agradecimientos la Corporación Nacional Forestal (CONAF) y el Departamento de Áreas Silvestres Protegidas de la región de Los Lagos por las facilidades y permisos de investigación otorgados. Este trabajo fue apoyado por las siguientes instituciones: US National Science Foundation (0823380 and 0917697), US Forest Service Pacific Northwest Research Station, Dirección de Investigación y Desarrollo (DID), y el Centro de Ciencia del Clima y la Resiliencia (CR)2 (CONICYT/FONDAP/15110009).

\section{LITERATURA CITADA}

Arno, S. F., \& Sneck, K. M. (1977). A method for determining fire history in coniferous forests of the mountain west. USDA Forest Service Intermountain Forest and Range Experiment Station, General Technical Report, INT- 42.

Donoso, C. (1993). Estructura, variación y dinámica de bosques templados de Chile y Argentina. Ecología Forestal. Santiago, Chile: Editorial Universitaria.

Duncan, R. P. (1989). An evaluation of errors in tree age estimates based on increment cores in kahikatea (Dacrycarpus dacrydioides). New Zealand Natural Sciences, 16, 31-37.

Franklin, J. F., Lindenmayer, D. B., MacMahon, J. A., McKee, A., Magnuson, J. J., Perry, D. A., Waide, R. B. \& Foster, D. R. (2000). Threads of continuity: ecosystem disturbance, recovery, and the theory of biological legacies. Conservation Biology in Practice, 1(1), 8-16.

Godoy, R., Oyarzún, C., \& Bahamondes, J. (1999). Flujos hidroquímicos en un bosque de Nothofagus pumilio en el Parque Nacional Puyehue, sur de Chile. Revista Chilena de Historia Natural, 72, 579-594.

González-Ferrán, O. (1995). Volcanes de Chile. Santiago, Chile: Instituto Geográfico Militar.
González, M. E., Amoroso, M., Lara, A., Veblen, T. T., Donoso, C., Kitzberger, T., Mundo, I.,... Promis, A. (2014). Ecología de disturbios y su influencia en los bosques templados de Chile y Argentina. En C. Donoso, M.E. González, \& A. Lara (Eds.), Ecología Forestal: Bases para el Manejo Sustentable y Conservación de los Bosques Nativos de Chile (pp. 411-502). Valdivia: Ediciones Universidad Austral de Chile.

Kitzberger, T., \& Veblen, T. T. (2003). Influences of climate on fire in northern Patagonia, Argentina. In T. T. Veblen, W. L. Baker, G. Montenegro, T. W. Swetnam (Eds.), Fire Regimes and Climatic Change in Temperate Ecosystems of the Western Americas. (pp. 411-502). New York: Springer-Verlag.

Lara, L. E., Moreno, H., Naranjo, J. A., Matthews, S., \& Pérez de Arce, C. (2006). Magmatic evolution of the Puyehue- Cordón Caulle Volcanic Complex (40 $\mathrm{S})$, Southern Andean Volcanic Zone: From shield to unusual rhyolitic fissure volcanism. Journal of Volcanology and Geothermal Research, 157, 343-366.

Montiel, M. (2014). Efecto de disturbios catastróficos sobre el establecimiento y crecimiento radial de especies del género Nothofagus en bosques afectados por la caída de tefra, Parque Nacional Puyehue. Tesis Magíster en Ciencias, mención Recursos Forestales. Valdivia, Chile: Facultad de Ciencias Forestales y Recursos Naturales, Universidad Austral de Chile.

Novoa-Melson, R. (2013). Efectos de la caída de tefra en bosques de Nothofagus pumilio, post erupción del Complejo Volcánico Puyehue-Cordón Caulle, Puyehue, Chile. Tesis Magíster en Ciencias, mención Recursos Forestales. Valdivia, Chile: Facultad de Ciencias Forestales y Recursos Naturales, Universidad Austral de Chile.

Stokes, M., \& Smiley, T. (1968). An introduction to tree-ring dating. Chicago: University of Chicago Press.

Veblen, T. T. (1985). Stand dynamics in Chilean Nothofagus forests. En S. T. A. Pickett, \& P. S. White (Eds.), The Ecology of Natural 
Disturbance and Patch Dynamics (pp. 3551). New York: Academic Press.

Vogel, A. (1996). Beobachtungen zur Regeneration der Vegetation nach Ascheeruption am Hudson-Vulkan im südlichen Chile. En Holtmeier, F. K. (Ed.), Beiträge aus den Arbeitsgebieten am Institut für Landschaftsökologie (pp. 3-11). Arbeiten aus dem Institut für Landschaftsökologie 1.

Wright, C., \& Mella, A. (1963). Modifications to the soil pattern of South-Central Chile resulting from seismic and associated phenomena during the period May to August 1960. Bulletin of the Seismological Society of America, 53,1367-1402. 
